\title{
Integrating Autonomic Grid Components and Process-Driven Business Applications
}

\author{
Thomas Weigold ${ }^{1}$, Marco Aldinucci $^{2}$, Marco Danelutto $^{3}$, and Vladimir Getov ${ }^{4}$ \\ 1 IBM Zurich Research Lab., Zurich, Switzerland \\ twe@zurich.ibm.com \\ 2 Computer Science Dept., University of Torino, Italy \\ aldinuc@di.unito.it \\ 3 Computer Science Dept., University of Pisa, Italy \\ marcod@di.unipi.it \\ 4 School of Electronics and Computer Science, University of Westminster, London, \\ U.K. \\ V.S.Getov@westminster.ac.uk
}

\begin{abstract}
Today's business applications are increasingly process driven, meaning that the main application logic is executed by a dedicate process engine. In addition, component-oriented software development has been attracting attention for building complex distributed applications. In this paper we present the experiences gained from building a process-driven biometric identification application which makes use of Grid infrastructures via the Grid Component Model (GCM). GCM, besides guaranteeing access to Grid resources, supports autonomic management of notable parallel composite components. This feature is exploited within our biometric identification application to ensure real time identification of fingerprints. Therefore, we briefly introduce the GCM framework and the process engine used, and we describe the implementation of the application using autonomic GCM components. Finally, we summarize the results, experiences, and lessons learned focusing on the integration of autonomic GCM components and the process-driven approach.
\end{abstract}

Key words: autonomic computing, components, parallel applications, distributed applications, process-driven applications

\section{Introduction}

Today's businesses are increasingly process driven. Ideally, all actions within an enterprise are explicitly defined as processes with the goal to improve control, flexibility, and effectiveness of delivering customer value. Additionally, business processes are oftentimes supported or even fully implemented by software applications [1]. In many cases, the business processes are turned into software such that they are hidden in the application's source code. However, there is a trend towards separating the main business logic from the functional code such that the resulting applications become more transparent and more flexible. The approach is to embed a so-called process engine into the application, 
which then executes process definitions representing the main control logic of the application. Functional code is then triggered from the process engine in accordance with the process definition. Such applications are called process-driven or workflow-driven applications $[2,3,4]$. The main advantages of this approach are the fact that the application logic can be modified without re-compiling the application, even at runtime, the business logic is more evident, and monitoring features of the process engine can be explored.

Besides the trend towards process-driven applications, enterprises seek ways to benefit from resources available from computing Grids/Clouds, in particular in all those cases were parallel computing is required to guarantee fair performances. The development of grid applications is not an easy task, however. Grid architectures present peculiar features such as dynamicity, heterogeneity and non exclusive access to resources, that require substantial effort to be suitably handled; furthermore, this effort is in addition to the normal effort required to develop efficient parallel/distributed applications. Within the plethora of programming environments targeting Grids, GCM (the Grid Component Model developed within CoreGRID [5] and whose reference implementation has been provided by GridCOMP) [6]) supports Grid programmers in designing parallel/distributed grid applications. In particular, GCM provides pre-defined composite components modelling standard parallel/distributed computation patterns that users can instantiate just providing the components implementing the sequential computations involved in the parallel pattern. In addition, these pre-defined composite components implement proper autonomic managers that completely take care of non functional aspects related to application execution according to what is specified in user supplied contracts.

In this work, we discuss a process-driven application, which makes use of GCM autonomic components to solve the problem of large-scale biometric identification[7], that has been developed as part of the activities of the GridCOMP project [6]. In particular, we discuss how process-driven application development exploits the autonomic features provided by the underlying Grid software as well as the results, experiences, and lessons learned during application development focusing on the integration of autonomic GCM components and the process-driven approach.

The paper is organized as follows: Sec. 2 introduces GCM and Behavioural Skeletons (BS), i.e. the autonomic composite components modelling notable parallel/distributed patterns within GCM. Sec. 3 introduces the process engine used to implement the biometric identification application discussed in Sec. 4 on top of GCM/BS. Eventually, Sec. 5 discusses the overall results achieved and Sec. 6 drafts the conclusions of the paper.

\section{The GCM framework}

The Grid Component Model (GCM) is a component model explicitly designed to support component-based autonomic applications in distributed contexts [5]. The main features of this component model can be summarised as follows: 
- Hierarchical: GCM components can be composed in a hierarchical way in composite components. Composite components are first class components and they are not distinguishable from non composite components at the user level. Hierarchical composition greatly improves the expressive power of the component model and is inherited by GCM from the Fractal component model [8].

- Structured: In addition to standard intra-component interaction mechanisms (use/provide ports [9]) GCM allows components to interact through collective ports modelling common structured parallel computation communication patterns. These patterns include broadcast, multicast, scatter and gather communications operating on collections of components. Also, GCM provides data and stream ports, modelling access to shared data encapsulated into components and data flow streams. All these additional port types, not present in other well known component models, increase the possibilities offered to the component system user for developing efficient parallel component applications.

- Autonomic: GCM specifically supports implementing autonomic components in two distinct ways: by supporting the implementation of user defined component controllers and by providing behavioural skeletons. Component controllers can be programmed in the component membrane (the membrane concept, as the place where component control activities take place, is inherited from Fractal [8]) and controllers can be components themselves. This provides a substantial support to the development of reusable autonomic controllers. Behavioural skeletons, thoroughly discussed in Sec. 2.1, are composite GCM components modelling notable parallel/distributed computation patterns and supporting autonomic managers, i.e. components taking care of non functional concerns affecting parallel computation.

Due to the presence of controllers and autonomic managers, GCM components implement two distinct kinds of interfaces: functional and non-functional ones. The functional interfaces host those ports concerned with the implementation of the functional features of the component. The non-functional interfaces host the ports related to controllers and autonomic managers. These ports are the ones actually supporting the component management activity in the implementation of the non-functional features, i.e. those features contributing to the efficiency of the component in obtaining the expected (functional) results but not directly involved in result computation.

GCM has been designed within the Programming Model Institute [10] in CoreGRID [11] and a reference implementation of the component model has been developed within the GridCOMP project [6]. Within the same GridCOMP project, a Grid Integrated Development Environment (GIDE) has been developed to support development and maintenance of GCM programs. 


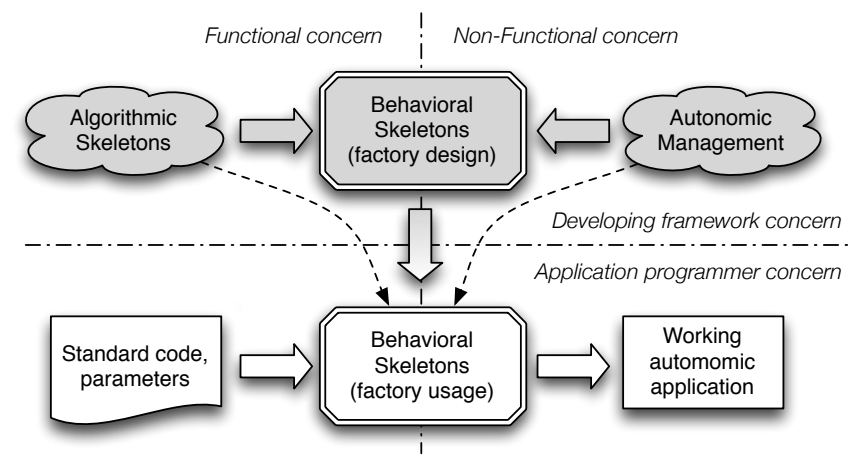

Fig. 1. Behavioural skeleton rationale

\subsection{Behavioural Skeletons}

Behavioural skeletons represent a specialisation of the algorithmic skeleton concept for component management [12]. Algorithmic skeletons have been traditionally used as a vehicle to provide efficient implementation templates of parallel paradigms. Behavioural skeletons, as algorithmic skeletons, represent patterns of parallel computations (which are expressed in GCM as graphs of components), but in addition they exploit the inherent skeleton semantics to design sound self-management schemes of parallel components.

Behavioural skeletons are composed of an algorithmic skeleton together with an autonomic manager (see Fig. 1). They provide the programmer with a component that can be turned into a running application by providing the code parameters needed to instantiate the algorithmic skeleton parameters (e.g. the code of the different stages in a pipeline or the code of the worker in a task farm) plus some kind of Service Level Agreement (SLA, e.g. the expected parallelism degree or the expected throughput of the application). The code parameters are used to build the actual code run on the target parallel/distributed architecture, while the SLA is used by the autonomic manager that will take care of ensuring this SLA (best effort) while the application is being computed.

The choice of the skeleton to be used as well as the code parameters provided to instantiate the behavioural skeleton are functional concerns: they only depend on what has to be computed (i.e. on the application at hand) and on the qualitative parallelism exploitation pattern the programmer wants to exploit. The autonomic management itself is a non-functional concern. The self-management and self-tuning activities taking place in the manager to ensure user supplied SLA both depend on the application structure (the one defined by the algorithmic skeleton) and on the target architecture at hand. The implementation of both the algorithmic skeleton and the autonomic manager is in the charge of the "system" programmer, i.e. the one providing the behavioural skeleton framework to the application user.

In the programming model provided by behavioural skeletons, the application programmers are in charge of picking up a behavioural skeleton (or a composition 
of behavioural skeletons) among those available and of providing the corresponding parameters and SLA. The system, and in particular the autonomic managers of the behavioural skeletons instantiated by the application programmer, are in charge of performing all those activities needed to ensure the user supplied SLA. These activities, in turn, may include varying some implementation parameters (e.g. the parallelism degree, the kind of communication protocol used among different parallel entities or scheduling/mapping of the parallel activities to the target processing elements) as well as changing the behavioural skeleton (composition) chosen by the application programmer (e.g. using "under the hoods" an equivalent, but more efficient (with respect to the target architecture and user supplied SLA) behavioural skeleton (composition)).

Autonomic management of non-functional concerns is based on the concurrent execution (with respect to the application "business logic") of a basic control loop such as that shown in Fig. 2. In the monitor phase, the application behaviour is observed, then in the analyse and plan phases the observed behaviour is examined to discover possible malfunctioning and corrective actions are planned. The corrective actions are usually taken from a library of known actions and the chosen action is determined by the result of the analysis phase. Finally, the actions planned are applied to the application during the execute phase $[13,14,15,16,17,18]$.

Currently, two kind of behavioural skeletons are implemented in GCM: a task farm BS and a data parallel BS (see Fig. 3). The former models embarrassingly parallel computations processing independent items $x_{i}$ of an input stream to obtain items $f\left(x_{i}\right)$ of the corresponding output stream. The latter models data parallel computations by computing for each item of the input stream $x_{i}$ an item $f\left(x_{i}, D\right)$ of the corresponding output stream, where $D$ represents a read only data structure and the result of $f\left(x_{i}, D\right)$ can be computed as a map of some function $f^{\prime}\left(x_{i}\right)$ on all the items of $D$ followed by a reduce of the different $f^{\prime}\left(x_{i}, D_{j}\right)$ with an associative and commutative operator $g$.

Both BS implement an AM taking care of the performance of the parallel computation at hand. In particular, the AM may ensure contracts stating the expected service time (or throughput, i.e. the time between the delivery of two

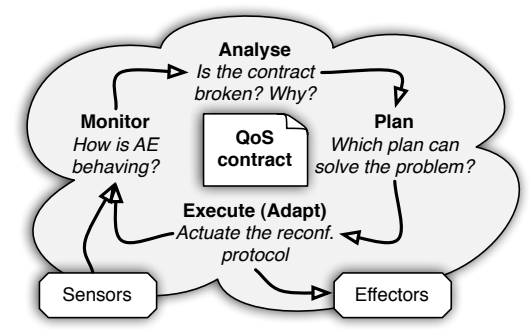

Fig. 2. The classical control loop implemented within Autonomic Managers in GCM Behavioural Skeletons 


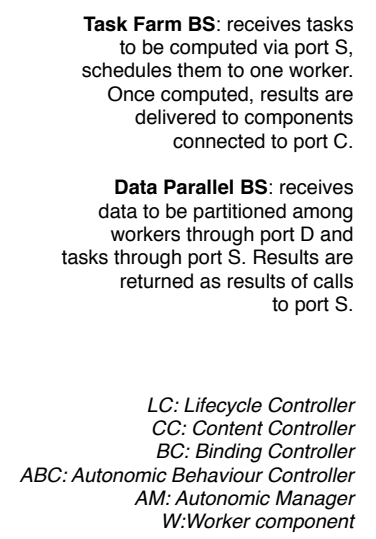

Task Farm BS

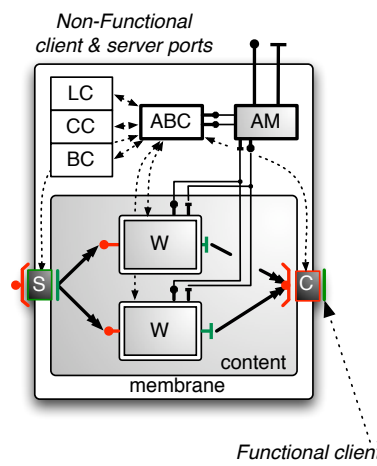

Data Parallel BS

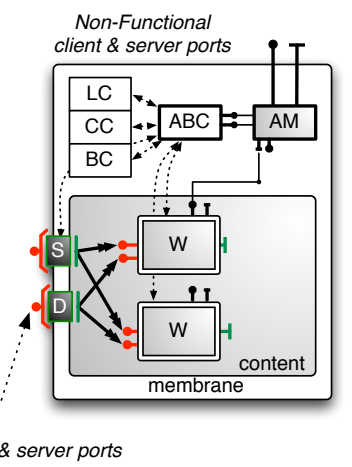

Fig. 3. Behavioural skeletons currently implemented in GCM

consecutive items on the output stream) of the BS (both task farm and data parallel BS) or the expected partition size of data structure $D$ (data parallel BS only). Currently, the contracts must be supplied to the BS AMs through the BS non functional ports as a(n ASCII string hosting a) set of JBoss rules defined in terms of the operations provided by the $\mathrm{ABC}$ controller bean. In fact, the AM control loop is implemented by running an instance of the JBoss business rule engine at regular intervals of time. At each time interval, all the pre-condition-action rules supplied to the AM are evaluated and those that turn out to be fireable (e.g. whose with the pre-condition holding true) are executed ordered by priority (or salience according to JBoss jargon). The pre-conditions are evaluated using values provided by the monitoring system implemented in the ABC controller beans, actually. The period used to run the JBoss engine is determined in such a way it is neither too fast (reacting when it was not the case to react to small changes in the system, thus increasing overhead to the autonomic management) nor too slow (poorly reacting to actual changes in the system, thus decreasing efficiency of autonomic management).

Current AMs manage the contracts varying the parallelism degree of the BS, i.e. the number of worker instances actually used to implement the BS. The variation of the number of worker instances happens adding/removing a fixed amount of workers. This fixed amount is a BS user configurable constant $\left(\Delta_{w}\right)$. Rules supplied to the AM in the BS also consist in specific rules avoiding to perform (probably) useless adaptations (e.g. avoiding to adapt BS parallelism degree immediately after another adaptation took place) as well as rules default actions basically only taking care of updating monitored values when no other, more significant actions turn out to be fireable. 


\section{The ePVM Process Engine}

The embeddable Process Virtual Machine (ePVM) is a research prototype process engine [4] basically built upon two core concepts. Firstly, a process model which is rooted in the theoretical framework of communicating extended finite state machines (CEFSM). Secondly, whereas many efforts have been made to create the ultimate process language, ePVM provides in contrast a low-level run-time environment based on a JavaScript interpreter where higher-level domain specific process languages can be mapped to.

The idea of ePVM can be considered to follow a bottom-up or micro-kernel type of approach for building process-driven applications, Business Process Management Systems (BPM), or workflow systems. This means that ePVM is a basic framework for building such systems rather than a complete off-the-shelf application that can run stand-alone. It consists of a library including a lightweight, generic, and easily programmable process execution engine. Lightweight hereby means that the engine is small in size and imposes minimum requirements on its environment, namely the host application it is embedded in. ePVM has its own process model resembling networks of communicating state machines running in parallel, which makes it an inherently asynchronous, event-driven run-time system. Every state machine is implemented by one JavaScript function, has an associated thread executing it, has a state object which is passed every time the function is invoked, and can communicate with other processes as well as entities external to the process engine via some messaging system. An arbitrary number of external entities, so-called host processes, can be attached to the engine to become visible for ePVM processes. The ePVM programming model based on the theory of CEFSM combines the simplicity of JavaScript with an easy and powerful way of defining complex concurrent business processes. More details can be found in [4].

\section{Process-Driven Distributed Biometric Identification}

In recent years biometric methods for verification and identification of people have become very popular. Applications span from governmental projects like border control or criminal identification to civil purposes such as e-commerce, network access, or transport. Frequently, biometric verification is used to authenticate people meaning that a 1:1 match operation of a claimed identity to the one stored in a reference system is carried out. In an identification system, however, the complexity is much higher. Here, a person's identity is to be determined solely on biometric information, which requires matching the live scan of his biometrics against all enrolled (known) identities. Such a 1:N match operation can be quite time-consuming making it unsuitable for real-time applications. In order to tackle this challenge, a distributed biometric identification system (BIS), which can work on a large user population of up to millions of individuals, has been developed. It is based on fingerprint biometrics and allows real-time 
identification within a few seconds period by taking advantage of the Grid, in particular via GCM components.

\subsection{Application Architecture}

The BIS can be considered a process-driven application, as it is centrally driven by the ePVM process engine. Fig. 4 outlines its high-level architectural design.

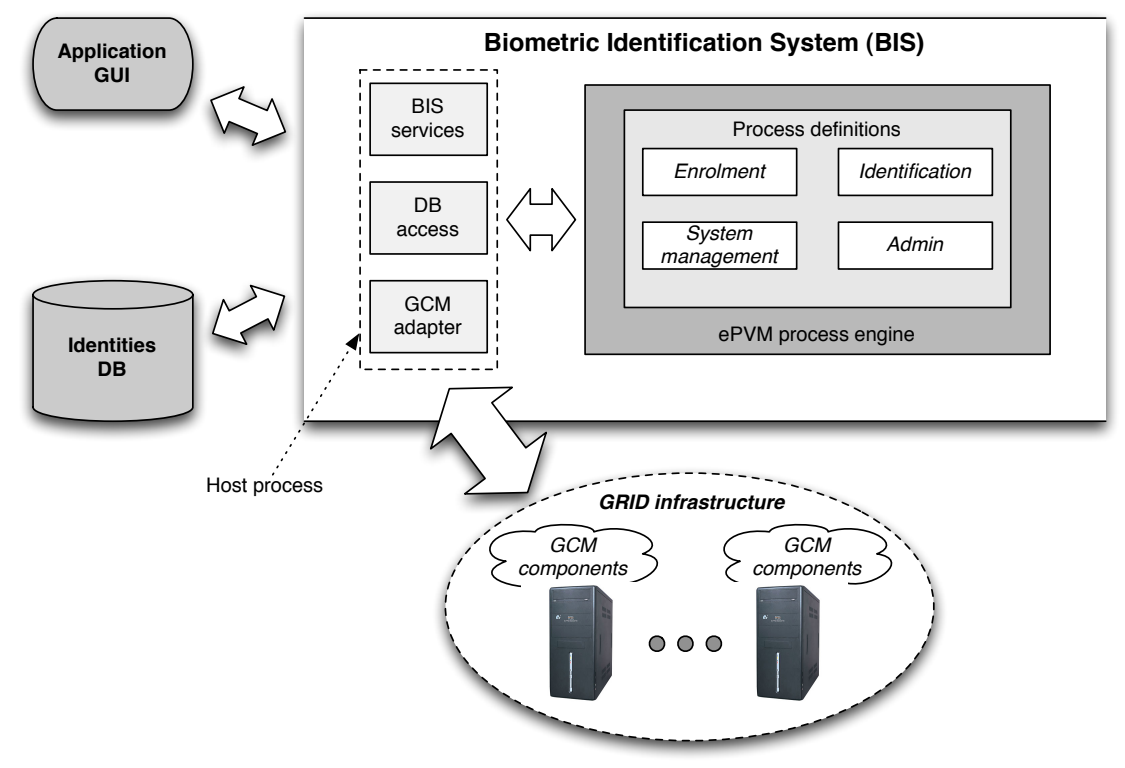

Fig. 4. BIS high-level architecture

A number of ePVM process definitions describing the main control flow for operations such as starting up the system or identifying a person are loaded into the process engine. These processes co-operate with external entities such as the GUI, the database (DB) of known identities, and the distributed GCM component system via a number of host processes to implement the overall functionality of the BIS.

\subsection{Process-Engine/GCM Interfacing}

The actual distributed fingerprint matching functionality is implemented via a set of GCM components deployed within a Grid/Cloud infrastructure as indicated in Fig. 4. Processes running within the process engine must be able to create, deploy, configure and interact with these components. For this purpose, a dedicated host process named GCM adapter (c.f. Fig. 4) has been developed, 
which receives messages from ePVM process instances, turns these messages into method invocations on GCM framework methods or GCM components, and generates appropriate reply messages returned to ePVM. The GCM adapter represents the main interface between ePVM and GCM. As ePVM process definitions are implemented in JavaScript and the GCM framework is available as a Java library, the GCM adapter essentially converts between JavaScript messages and Java method invocations.

An alternative option would have been to export the GCM components as Web services, as supported by the GCM implementation, and invoke them from within the GCM adapter. However, this would have increased the number of required type conversions going from Java Script over SOAP to Java and vice versa. Also, the GCM framework only supports exporting GCM components as Web services. Other framework services, for example, functionality for deployment and component creation, cannot be turned into Web services automatically. Finally, the ePVM process engine does not necessarily require working on Web Services level like, for instance, process engines based on the Business Process Execution Language (BPEL). Consequently, we decided not to use Web services as interfaces between the process engine and GCM.

The functionality provided by the GCM adapter includes:

- Activate a given GCM deployment descriptor to start the nodes available in the Grid.

- Modify architecture description language (ADL) files describing the GCM components used.

- Create GCM components within the Grid.

- Invoke methods on GCM components, for example, to configure the quality of service (QoS) contract, distribute the DB of known identities, or submit the biometrics of a person for identification.

The GCM adapter is triggered by ePVM process instances to implement the overall application logic. As an example, the activity flow chart shown in Fig. 5 illustrates the control logic implemented within an ePVM processes as it is executed during BIS initialization. For each of the activities a message is being sent to a host adapter which implements the functionality. Some of the activities execute in parallel, for instance, activity 1.1 to 1.3 , some are sequential.

\subsection{Using Autonomic GCM Components}

The problem of biometric identification can be considered a search problem where the compare function is a biometric matching algorithm, here fingerprint matching. To distribute the problem within a Grid infrastructure, the DB of known identities needs to be distributed such that each computing node in the Grid receives a partition of the overall DB and can match a given identity against

this partition. The time spent in matching the given identity against the local portion of the database is clearly proportional to the size of this local DB portion. Therefore, considering that the distribution of the DB among the grid 


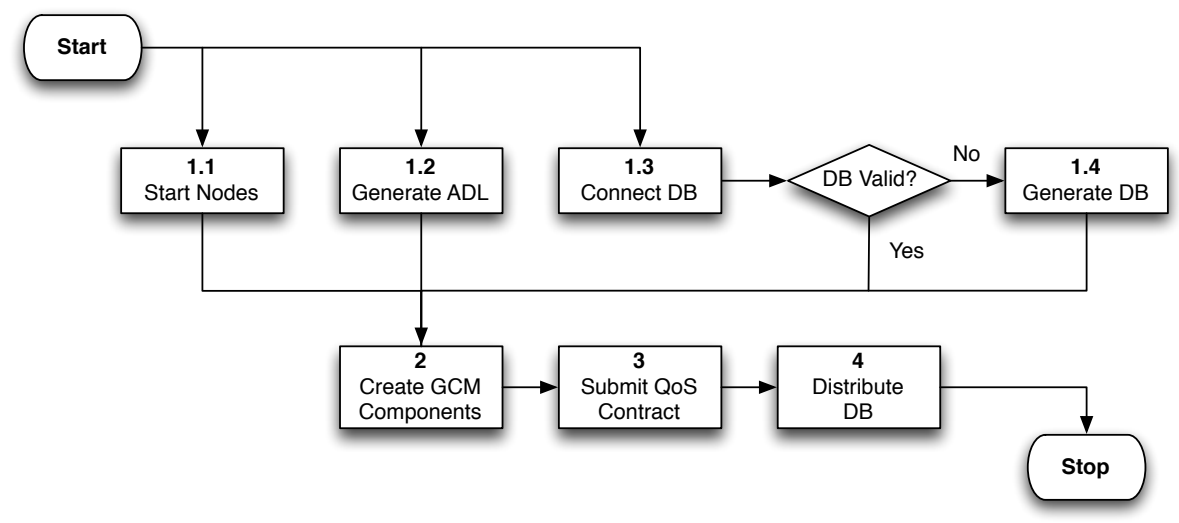

Fig. 5. BIS initialization process flow

nodes is performed once and for all, and considering negligible the time spent to broadcast the fingerprint that has to be matched with those in the distributed database, the ability to perform fingerprint matching in real time roughly depends on the ability to distribute local portions of the database small enough to allow real time matching of the broadcasted fingerprint. More precisely, the time spent in matching a single fingerprint against the local database also depends on the computing power and on the load of the machine used to perform the matching. The machine power and the local database sizes are somehow static properties. The load of the machine is instead a dynamic property. Thus, in order to keep the matching time perceived by the application user within a given range (i.e. satisfying a given service level agreement (SLA) or performance contract), our BIS application should i) properly dimension the number of distributed resources used to host database portions and ii) dynamically adapt to the varying load of the grid resources involved in such a way a user supplied performance contract (such as match fingerprint in less than 30 secs) is ensured. Both features are supported within the GCM Behavioural skeletons presented in Sec. 2.1: if the user instantiates a Behavioural skeleton to implement the BIS search process, and if he/she provides a contract stating the expected latency of the fingerprint matching process, the AM of the behavioural skeleton will start with a predefined number of workers (i.e. a predefined parallelism degree) and then adapt this number to achieve the matching latency adding (removing) workers from the BS composite component. In case of overload of some of the resources used in the matching, the AM of the behavioural skeleton will also manage to increase the number of resources recruited to the parallel matching, in such a way the contract can be ensured again. In this case, the recruitment of a new processing resource induces a physical redistribution of the database among the resources. This redistribution is completely implemented/managed by the behavioural skeleton AM. 
In order to implement our BIS application, we used a data parallel (DP) behavioural skeleton. Referring to Fig. 3 (right), the DP skeleton is a composite component which includes an autonomic behaviour controller (ABC) and an autonomic manager (AM). The AM periodically evaluates certain monitored properties of the skeleton to ensure that a given QoS contract is satisfied. If this is not the case, it triggers appropriate reconfiguration operations provided by the ABC. To apply the DP skeleton for our application scenario, it must be parametrised with a worker component and a QoS contract. The worker component, here named IDMatcher, implements the actual fingerprint matching functionality and the skeleton allocates one instance of this worker component per node. The QoS contract consists of a set of rules interpreted by the JBoss Drools rule engine.

For our BIS prototype we chose to implement a QoS contract requiring to keep the partition size of the workers constant, independently of the size of the database presented to the BS through port $\mathrm{D}$. The contract is provided before starting the computation through the non functional server port attached to the BS AM. The AM, in this case, adds or removes workers from the BS in case the partition size exceeds or is less than the value supplied by the user within the contract provided through the non functional BS ports.

Before identification requests can be processed, the identity DB is distributed across the worker components using port $\mathrm{D}$. As a consequence, the $\mathrm{DB}$ is partitioned on the inner $\mathrm{W}$ components. The identity DB holds information such as name, address, and fingerprints of all enrolled (known) people.

Once the skeleton has been initialized, identification requests can be submitted via the second port provided by the BS, port S, the so-called broadcast port. Fingerprints of a person to be identified are broadcasted via this port to all worker components and each worker matches them against its partition of the DB. Results are returned synchronously via method return values.

If the $\mathrm{AM}$ triggers reconfiguration via the $\mathrm{ABC}$, for example, to increase the number of worker components, the AM collects all DB partitions from the workers, modifies the number of workers, and finally redistributes the DB to the workers. This way the DB is redistributed during each reconfiguration operation.

The submission of the contract through non functional interfaces, the DB through $\mathrm{BS}$ port $\mathrm{D}$, and the fingerprints to be matched through port $\mathrm{S}$ are all interactions with the GCM BS triggered by ePVM processes via the GCM adapter.

\subsection{Deployment and Component Creation}

When the BIS application is started, activation of the GCM deployment descriptor is triggered by the process engine as indicated in Fig. 5, activity 1.1. The GCM framework then defines virtual nodes, creates a mapping to real nodes, and starts JVMs on all of them. The DP skeleton uses virtual nodes listed in the descriptor for allocating worker components. Afterwards, when the initialization process reaches activity 2, the GCM component system is created according to the ADL files of the BS. 


\subsection{Application Monitoring}

Monitoring is one of the core features of every process engine and it is an important argument for using one when building an application. The ePVM engine supports monitoring processes by registering monitor objects for one or more process definitions. Furthermore, it can be specified which events shall be monitored. Available are a number of standard events such as a process instance being created, a message being processed, or a process becoming idle. Furthermore, custom events can be defined such that more fine-grained monitoring can be implemented, for example, multiple events can be triggered while a single message is processed.

In the BIS application, a monitor object is used to track the progress of ePVM process instances, for example, while the system initialization process is executed (c.f. Fig. 5). The monitor object is triggered by the process engine whenever activities start or finish and it updates the GUI to reflect the state of the system. Furthermore, it is desired to monitor the GCM component system with the goal to visualize AM actions and the number of workers used in the DP skeleton. A system administrator could observe this and, if required, trigger reconfiguration or add resources manually. For monitoring the skeleton, functionality provided by the GCM framework can be used. However, monitoring in GCM is based on a pull model where information about components and their states can be retrieved on request. On the contrary, the ePVM approach can be considered a push model where a monitor is registered and receives events. To integrate GCM monitoring with the event-driven paradigm applied in ePVM some adaptation is necessary. A first solution is to create a dedicated ePVM process which regularly retrieves information about the component system via the GCM API and creates events for the monitor object. A second solution is to instrument the component implementation to actively send events to an ePVM process. The first approach is more generic with respect to distribution, as the GCM framework handles remote method invocations required to query for component states automatically. The second approach is more efficient, as communication only takes place if an event to be monitored occurs. However, a component might not be able to easily communicate with the process engine if it is running on a remote machine, since the process engine itself is not a GCM component. In the BIS we used the first approach to implement monitoring the number of workers, as the workers are typically distributed. For monitoring AM actions, we use the second approach exploiting the fact that in our deployments the AM is always co-located with the process engine such that no remote communication is necessary.

In general, the requirement to monitor actions within the DP skeleton to some extend is contradictory to the idea of autonomic components. On one hand the goal of using the DP skeleton is to take advantage of its built-in functionality without taking care of the implementation details. On the other hand, we still want to be able to monitor certain internal details such as reconfiguration operations and the number of workers. From the perspective of the process-driven applications paradigm all important actions which shall be monitored should be 
centrally controlled by the process-engine. However, in real-world applications a trade-off between central process control and autonomy must be made.

\subsection{Automatic Futures vs. Message Passing}

When integrating process-engines and distributed computing frameworks, it is very important to be aware of their communication and synchronization paradigms. The GCM framework is based on Java RMI and implements the concept of automatic futures [19]. This means that method invocations always return immediately, whereas results which are not yet available are represented by so-called future objects. Program execution is then blocked automatically if a future object is being accessed as long as the value represented is not yet available. The goal is to ease parallel programming by hiding synchronization details within a meta object protocol implemented in GCM. The ePVM process engine, however, uses message passing for communication and synchronization between concurrent control flows. If these two paradigms are interweaved, as it is the case in the BIS application, process flows can easily become distorted. For example, if a process definition assigns two activities to be carried out sequentially (c.f. activity 2 and 3 in Fig. 5), it must be ensured that no more future objects resulting from the first activity exist before the second is triggered.

This issue becomes obvious when an identification process is triggered within the BIS. In this case, an ePVM process sends a message to the GCM adapter including fingerprints of a person to be identified. The GCM adapter forwards this information to the component system by invoking the broadcast interface of the DP skeleton (port S, Fig. 3). This interface is a so-called collective interface, which turns one method invocation into $\mathrm{N}$ method invocations on all the bound IDMatcher components to broadcast the identification request. The return value is a list of result objects, one from each IDMatcher component. When the interface is invoked, it immediately returns a list of future objects, which at the beginning are all unavailable and then by-and-by become available as the IDMatcher components return their results. It is important that the GCM adapter waits for the futures to become available and generates messages to be returned to the ePVM process instance accordingly. It must not report the identification as completed before all futures are available. Effectively, the GCM adapter retracts automatic synchronization in order to make the actual progress visible to the process engine, which must to be informed whenever an IDMatcher component has searched its part of the DB. Obviously, converting from one paradigm into the other must be handled with care as the semantics of the process definitions can be broken due to delayed synchronization within GCM.

\subsection{Integrated Development}

On one hand, the advanced features offered by both technologies, the process engine and the GCM framework, significantly reduce the development effort required for the BIS. On the other hand, it requires handling a large number of 
different development artifacts including plain Java code, JavaScript process definitions, XML deployment descriptors and ADL definitions, and JBoss Drools rule files. As the process engine does not mandate the use of high-level modelling tools, developers can use the Java/JavaScript toolset of their choice. For GCM development, the Grid IDE (GIDE) [20] is available, which consists of a set of plugins to the famous Eclipse development environment. It also includes support for graphical GCM component composition and ADL code generation. Consequently, all artifacts can be developed within Eclipse with appropriate plugins installed. This reduces the complexity to a manageable level, such that once the knowledge about both technologies is available, integration work can be carried out smoothly.

\section{Results, Experiences, and Lessons Learned}

The primary result of this work is the fully functional prototype of the BIS application, which acts as a use case demo for the process engine as well as for the GCM framework. Additional results have been gained by critically evaluating the application and experimenting with it. Firstly, it has been successfully deployed on various hardware platforms ranging from one multi-core $\mathrm{PC}$ to heterogeneous sets of clusters as provided by the Grid5000 project [21]. Switching hardware platforms did not require changing a single line of functional code, only the infrastructure part of the XML deployment descriptor required modification. The strict separation of concerns and the autonomic functionality implemented within the GCM framework have turned out to be the main factors leading to this flexibility. The former ensures that resources are never directly referenced in the source code while the latter provides autonomic adaptation to the performance properties of the hardware in use.

Secondly, functionality and autonomic behaviour of the application has been verified using Grid5000. The BIS has been started using 50 workers (one per node), a DB holding 50000 identities (approx. $400 \mathrm{MB}$ ), and a QoS contract mandating a partition size of 1000 identities/worker. At runtime, the contract has been updated to $800( \pm 10 \%)$ identities/worker. Thereupon, the AM has successfully detected 7 contract violations and each time reconfigured the DP skeleton by adding one additional worker until a partition size of 877 identities/worker was reached at 57 workers/nodes. During this experiment, every reconfiguration operation took about 9 seconds in which the complete DB has been redistributed (from the node hosting the whole database to the nodes hosting the workers of the data parallel $\mathrm{BS}$ ) by the $\mathrm{ABC}$. When identification requests where issued during reconfiguration, they where queued automatically by the skeleton and processed as soon as reconfiguration was completed. For the given DB size, each identification request required around 10 seconds to be processed. This means that each reconfiguration operation roughly decreases the throughput of the BIS by one identification for any given timeframe. Therefore, if the BIS is used in a very dynamic environment requiring frequent reconfiguration, the number of occurrences of reconfigurations may be sensibly reduced by adopting 
more aggressive parallelism degree variation policies, in such a way the overall overhead is reduced. Such more aggressive policies at the moment consist in varying the constant $\Delta_{w}$ that defines the number of workers to be added/removed when reconfiguring the parallelism degree of a BS. As shown in [17], the evaluation of such $\Delta_{w}$ can also take in account the overhead and the delay of the reconfiguration itself by using historical data. In the BS/GCM framework we are currently investigating the possibility to use a kind of exponential backoff increase/decrease protocol. All those cases, of course, rely on the possibility to effectively monitor the increase/decrease achieved in the BS performance as a consequence of the parallelism degree adaptation.

Finally, evaluating the application's source code, including the deployment descriptor required to run on 50 nodes of Grid5000, unveiled the source code breakdown illustrated in Fig. 6. The functional code mainly includes the host processes (c.f. Fig. 4) providing DB access, the GUI functionality, and the interfacing to the GCM components. Its absolute size is about 2500 lines of code, which is very small considering the the overall functionality provided by the application. This is due to the fact that the GCM framework provides all the functionality for distribution and autonomicity. Implementing this functionality from scratch not using GCM would have been significantly more effort. In particular, adding autonomic control to an application is virtually effortless if a matching behavioural skeleton is available. Only the QoS contract must be provided and a few non-functional interfaces used by the controller must be implemented within the worker component. In case of the BIS application, only about 200 lines of code where necessary for that. Furthermore, it is to be noted that more than a quarter of the source code $(27 \%)$ consists of code interpreted at runtime. This code, including the deployment descriptor, the process definitions, the QoS contract, and the GCM component definitions, contains the main control logic and infrastructure definition of the application. As a result, the application can be adapted significantly without recompilation - a very important property required for operation in today's dynamic business environments. Hard-coding this part of the application would clearly decrease the applications flexibility as achieved through the combination of GCM and ePVM.

During application development, we have made a number of experiences with regards to the integration of process technology and the GCM framework. The interfacing between the two technologies went rather smoothly, since the ePVM engine is available as a Java library and it does not dictate the use of Web services. Also, the DP skeleton fits well to the given biometric identification problem. However, application monitoring turned out to be challenging. One must be aware that the idea behind components is hiding complexity and this can be a problem if component internals need to be monitored. The GCM framework supports querying the state of a component system, however, it does not support monitoring activities within components, for example, reconfiguration within a BS. Solving this problem by instrumenting component implementations (c.f. Sec. 4.5) requires comprehensive knowledge of the GCM framework. Furthermore, the monitoring support of GCM follows a pull model while process 


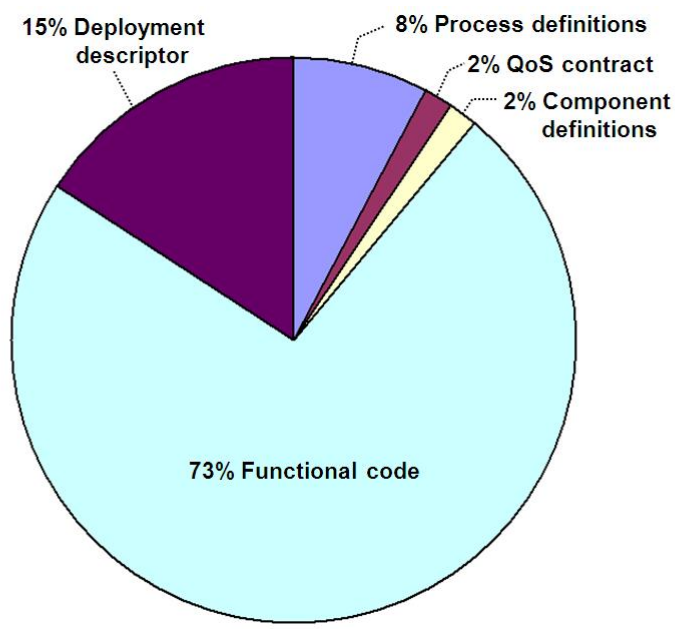

Fig. 6. BIS source code breakdown

engines are mostly event driven. Joining the two paradigms in a sensible way requires an extra effort and can have a performance impact. For example, regularly traversing component hierarchies to detect newly created components is not very efficient.

Another lesson we have learned is that the two different synchronization paradigms applied in GCM and ePVM can interfere if not handled with care. The concept of automatic futures implemented in the GCM framework follows the wait-by-necessity idea. This means that unavailable results are replaced by future objects such that synchronization is delayed as long as possible. Therefore, it must be carefully checked if results of activities within a process flow include one or more future objects before the next activity of a sequence is triggered, otherwise the process semantics can easily become distorted. In other words, if a GCM component returns an object it does not necessarily mean that all the related operations have completed.

Finally, we realized that working with the advanced features of both frameworks, ePVM and GCM, requires working with a large number of different development artifacts and acquiring related skills. The GIDE eases this to some extend and provides a jump start into GCM. Nevertheless, combining process technology with GCM allows producing extremely flexible and complex distributed applications with minimum effort.

\section{Conclusions}

Process-driven application development is increasingly gaining attention in the business environment. At the same time, software development frameworks for the Grid/Cloud are raising interest in the course of the Cloud computing wave. 
In this paper we have considered combining the two approaches to produce a process-driven distributed biometric identification system. In discussing the application we have made the following contributions:

- We provided a brief overview of the GCM framework, its support for autonomic components and behavioural skeletons, and the ePVM process engine.

- We described the architectural design and implementation of the processdriven biometric identification system utilizing the DP autonomic behavioural skeleton available in GCM.

- We presented the results, experiences, and lessons learned while integrating both technologies, the process engine and the GCM framework.

We believe that this use case application demonstrates that combining process technology and autonomic Grid/Cloud components represents a powerful approach for developing flexible distributed applications with minimum effort. Obviously, the application could have been developed without using GCM and ePVM. However, the development effort would have been much higher and the resulting application would have been less flexible due to the hard-coded application logic and autonomic strategy.

\section{References}

1. zur Muehlen, M.: Process-driven management information systems - combining data warehouses and workflow technology. In: Proc. of the 4th Intl. Conference on Electronic Commerce Research (ICECR-4), Dallas, TX, USA (2001) 550-556

2. Bukovics, B.: Pro WF: Windows Workflow in .NET 3.0. Apress (2007)

3. Faura, M.V., Baeyens, T.: The Process Virtual Machine. (2007) http://www. onjava.com/pub/a/onjava/2007/05/07/the-process-virtual-machine.html.

4. Weigold, T., Kramp, T., Buhler, P.: ePVM - an embeddable Process Virtual Machine. In: Proc. of the 31st Intl. Computer Software and Applications Conference (COMPSAC), Beijing, China (2007) 557-564

5. CoreGRID NoE deliverable series, Institute on Programming Model: Deliverable D.PM.04 - Basic Features of the Grid Component Model (assessed). (2007) http: //www. coregrid.net/mambo/images/stories/Deliverables/d.pm.04.pdf.

6. GridCOMP Project: Grid Programming with Components, An Advanced Component Platform for an Effective Invisible Grid (2008) http://gridcomp.ercim.org.

7. Weigold, T., Buhler, P., Thiyagalingam, J., Basukoski, A., Getov, V.: Advanced grid programming with components: A biometric identification case study. In: Proc. of the 32nd Intl. Computer Software and Applications Conference (COMPSAC), Turku, Finland, IEEE (2008) 401-408

8. ObjectWeb Consortium: The Fractal Component Model, Technical Specification. (2003)

9. Armstrong, R., Gannon, D., Geist, A., Keahey, K., Kohn, S., McInnes, L., Parker, S., Smolinski, B.: Toward a common component architecture for high performance scientific computing. In: Proc. of the 8th Intl. Symposium on High Performance Distributed Computing (HPDC'99). (1999)

10. CoreGRID NoE: Home page of the Institute on Programming model. (2009 - last accessed) http://www. coregrid.net/mambo/content/blogcategory/13/292/. 
11. CoreGRID NoE: Home page. (2009 - last accessed) http://www . coregrid.net.

12. Cole, M.: Bringing skeletons out of the closet: A pragmatic manifesto for skeletal parallel programming. Parallel Computing 30 (2004) 389-406

13. Kephart, J.O., Chess, D.M.: The vision of autonomic computing. IEEE Computer 36 (2003) 41-50

14. Danelutto, M.: QoS in parallel programming through application managers. In: Proc. of Intl. Euromicro PDP: Parallel Distributed and network-based Processing, Lugano, Switzerland, IEEE (2005) 282-289

15. Aldinucci, M., Danelutto, M.: Algorithmic skeletons meeting grids. Parallel Computing 32 (2006) 449-462

16. Aldinucci, M., Campa, S., Danelutto, M., Dazzi, P., Kilpatrick, P., Laforenza, D., Tonellotto, N.: Behavioural skeletons for component autonomic management on grids. In: CoreGRID Workshop on Grid Programming Model, Grid and P2P Systems Architecture, Grid Systems, Tools and Environments, Heraklion, Crete, Greece (2007)

17. Aldinucci, M., Danelutto, M., Kilpatrick, P.: Towards hierarchical management of autonomic components: a case study. In Didier El Baz, Tom Gross, F.S., ed.: Proc. of Intl. Euromicro PDP 2009: Parallel Distributed and network-based Processing, Weimar, Germany, IEEE (2009) 3-10

18. Aldinucci, M., Danelutto, M., Kilpatrick, P.: Autonomic management of nonfunctional concerns in distributed and parallel application programming. In: Proc. of Intl. Parallel \& Distributed Processing Symposium (IPDPS), Rome, Italy, IEEE (2009) 1-12

19. Caromel, D., Henrio, L.: A Theory of Distributed Object. Springer-Verlag (2005)

20. Basukoski, A., Getov, V., Thiyagalingam, J., Isaiadis, S.: Component-based development environment for grid systems: Design and implementation. In Danelutto, M., Frangopoulou, P., Getov, V., eds.: Making Grids Work. CoreGRID. Springer (2008) 119-128

21. The Grid5000 Project: An infrastructure distributed in 9 sites around France, for research in large-scale parallel and distributed systems (2008) http://www. grid5000.fr. 